\title{
CONSUMISMO EN COSTA RICA: UNA TIPOLOGÍA DE JÓVENES EN CONTEXTOS URBANOS DE EXCLUSIÓN SOCIAL
}

\author{
CONSUMERISM IN COSTA RICA: A TYPOLOGY OF YOUNG PEOPLE \\ IN URBAN CONTEXTS OF SOCIAL EXCLUSION
}

Francisco Hernández Ulloa

Recibido: 07/04/2018 - Aceptado: 23/09/2018

\begin{abstract}
Resumen
El proceso globalizador provocó cambios en las sociedades centroamericanas, entre los que destaca la emergencia del consumismo. La población joven es clave para este fenómeno, aunque aún no existe suficiente evidencia empírica al respecto. Precisamente, el presente artículo expone los principales resultados de una investigación que tuvo como objetivo analizar críticamente las relaciones entre consumismo y exclusión social y su afectación a personas jóvenes. Se generó una tipología mediante el análisis de conglomerados biétapicos (two-step cluster). Entre los hallazgos destaca la presencia de una heterogeneidad de juventudes en el territorio con dificultades de inserción laboral, agravada para las mujeres. Además, se evidencia la fragilidad de la pertenencia social simbólica generada por el consumismo, fenómeno que legitima las desigualdades existentes.
\end{abstract}

Palabras clave: Consumismo, exclusión social, juventudes, pertenencia social, fragilidad.

\begin{abstract}
The globalization process made changes in Central American societies, among which the emergence of consumerism stands out. The young population is key in this phenomenon, although there is still not enough empirical evidence in this regard. Precisely, this paper presents the main results of an investigation that aimed to critically analyze the relationships between consumerism and social exclusion and its impact on young people. A typology of young people was generated through the two-step clusters analysis. Among the findings is the presence of a heterogeneity of youth in the territory with labor insertion difficulties, aggravated for women. In addition, there is evidence of the fragility of symbolic social belonging generated by consumerism, a phenomenon that legitimizes existing inequalities.
\end{abstract}

Keywords: Consumerism, social exclusion, youth, social belonging, fragility. 


\section{Introducción ${ }^{1}$}

La conjugación de transformaciones acaecidas, tanto en el momento de modernización nacional y en la modernización globalizada, propició el escenario de emergencia de un fenómeno poco estudiado, el consumismo. Su intensificación durante el período globalizador resulta paradójica en un contexto de persistencia de la miseria y profundización de las desigualdades sociales en Centroamérica. Este escenario se caracteriza, entre otras cosas, por la integración de elites financieras regionales y la proliferación posterior de servicios financieros (León Araya; Zúñiga-Ramírez), nuevas pautas de consumo alimentadas por la masificación y comercialización internacional de mercancías (Calderón-Umaña), el rol central que adquirió la participación de personas jóvenes en esas nuevas dinámicas de consumo (Beirte Brealaey) y un tipo de transgresión delictiva propiciada por necesidades de satisfacción de consumo (Calderón-Umaña). Al mismo tiempo, la consolidación de este tipo de dinámicas de consumo abonó a la conformación de repertorios de legitimación de desigualdades (Lungo-Rodríguez), estos están siendo profundizados por la exclusión social en un escenario compartido en Centroamérica (LungoRodríguez, Pérez Sáinz y Mora-Salas). ${ }^{2}$

Con este telón de fondo, el presente artículo expone los principales resultados de una investigación que se propuso como objetivo analizar críticamente cómo se expresan las relaciones entre consumismo y exclusión social y de qué manera afecta a las personas jóvenes de 18 a 29 años del distrito de Los Guido, en el cantón de Desamparados. Así, el estudio se propuso comprender cómo se conjuga la exclusión social y el consumismo en contextos en que el control territorial por la disputa de micromercados de droga abre oportunidades de transgresión y posibilidades de victimización para las personas jóvenes habitantes de estos territorios.

El artículo se compone de cinco secciones, además de esta introducción. En el primer apartado se desarrolla el marco analítico para comprender exclusión social y consumismo como problemáticas sociológicas. En la segunda sección se profundiza sobre el escenario de emergencia del consumismo. En el tercer apartado se contextualiza el origen del distrito de Los Guido de Desamparados y las características que justifican su elección como delimitación espacial de estudio. En el cuarto apartado se exponen algunos apuntes metodológicos sobre la construcción de una tipología, realizada mediante el análisis de conglomerados bietápicos (two-step cluster), a partir de los datos de una encuesta a jóvenes. El quinto apartado revela los principales resultados de la investigación. El documento finaliza con una serie de reflexiones derivadas del análisis conjunto de las problemáticas.

\section{Construyendo una mirada analítica: Reflexiones teóricas sobre el consumismo}

La investigación sobre el consumismo tiene un desarrollo escueto, aunque sobresalen dos propuestas teóricas interesantes para su discusión. Por un lado, la de Zygmunt Bauman, quien define el consumismo como: 
Un tipo de acuerdo social que resulta de la reconversión de los deseos, ganas o anhelos humanos (si se quiere "neutrales" respecto del sistema) en la principal fuerza de impulso y operaciones de la sociedad desempeña un papel preponderante en los procesos individuales y grupales de autoidentificación, y en la selección y consecución de políticas de vida individuales (Bauman 47).

Frente a esta propuesta se pueden generar al menos dos cuestionamientos. Lo primero es que la conceptualización de Bauman adolece la ausencia de una problematización sobre el poder en las dinámicas consumistas. Esto se ve en el punto de partida del autor, pues no toma en cuenta la existencia de asimetrías gestadas en el mercado de trabajo, que resultan de procesos de desempoderamiento y a su vez, inciden en el acceso al consumo efectivo. Es decir, el acceso al trabajo se requiere para garantizar el consumo, por lo tanto, el consumismo no tiene características de un acuerdo social producto de deseos humanos neutrales, más bien resulta una consecuencia de las transformaciones inducidas por el capitalismo, donde el consumo se consolidó como un aspecto central de la economía. En Costa Rica, los sectores productivos dedicados a ofrecer bienes y servicios de consumo toman protagonismo a partir de la apertura comercial y la integración financiera regional en donde germina el consumismo (Torres Rivas, León Araya, Robles).

Si bien el consumismo se generalizó y adoptó un rol central en las relaciones sociales, esta centralidad acaece principalmente en un plano simbólico. Es decir, la centralidad está relacionada con un proceso de individualización, con lo cual se privilegia la participación de población económicamente aventajada. Esto ha dado paso a cambiar de una condición de individuo/ciudadano a individuo/consumidor, otorgando esa centralidad al consumo (García-Canclini).

Las posibilidades de acceso al consumo están reguladas por el acceso al trabajo, por lo tanto, este sigue teniendo un rol articulador y de fuerza de impulso y operaciones en la sociedad, pues la disputa por el excedente generado en los mercados básicos continúa siendo el meollo donde la distribución primaria acontece. El consumismo como mecanismo de individualización, agregaríamos a la propuesta de Bauman, se trata no solo de un consumo que satisface necesidades materiales, más bien, puede adquirir una significación simbólica muy alta y un poder de diferenciación social. ${ }^{3}$

Por otro lado, la propuesta de Andy Baker plantea que el consumo ha funcionado como un motor de la globalización y que, en América Latina, la apertura comercial ha tenido más beneficios que daños en el mercado laboral; concluye que los latinoamericanos piensan los problemas económicos actuales más como consumidores que como trabajadores. Para este autor "el auge del consumismo es la razón por la que las brechas de situación económica han crecido más débiles en la nueva era del mercado en América Latina" (Baker 266). Además, apunta como un logro que existan encuentros entre los grupos sociales en los espacios de consumo para la adquisición de bienes y servicios relacionados con estatus argumentando que "la globalización ha debilitado, más que 
fortalecido, los sentimientos de frustración causados por la insatisfacción de metas de consumo en los grupos de menor ingreso" (Baker 268). Por último, añade que el consumismo se ha democratizado y que la amplia gama de bienes de consumo de estatus (positional goods) diluye las diferencias entre los individuos, es decir, existe consenso alrededor del consumo (Baker).

Sobre esta perspectiva, en primer lugar, sostenemos el cuestionamiento planteado anteriormente a la postura del consumismo como consenso social, pues se ha señalado que en el plano simbólico el acceso amplio a bienes de consumo ya no es por sí mismo un diferenciador, más bien son las dinámicas propias del consumismo las que permiten crear e interpretar esas distinciones. En segundo lugar, la evidencia empírica, señala que los espacios de consumo forman parte de repertorios de legitimación de las desigualdades, es decir, que existe desencuentro y diferenciación por el acceso al consumo entre los grupos sociales (Lungo-Rodríguez 269). En tercer lugar, a pesar de que la democratización del consumo ha incorporado a los sectores subalternos, ha sido un proceso frágil debido a la volatilidad propia del consumo. El mayor peso sobre la carga simbólica que sobre la dimensión material de la satisfacción de necesidades, así como el alcance cada vez menos homogéneo, más segmentado y diferenciado a los bienes públicos, generan una incorporación diferenciada de los sectores subalternos al consumo básico (Pérez Sáinz, 2014).

De esta forma, el acceso a ciertos bienes de consumo puede otorgar identidad y reconocimiento, incluso puede darse en espacios de relativa homogeneidad; sin embargo, el carácter efímero de esta integración simbólica generada en las dinámicas consumistas depende de la capacidad de consumo de quienes aspiran ingresar en ese espacio de referenciación. ${ }^{4}$

Por lo tanto, el consumismo no elimina las dinámicas de clase que se dan en los mercados básicos, más bien otorga una visión distorsionada que coloca al consumo como el espacio donde se deben resolver esas desigualdades (Pérez Sáinz, 2014). En otras palabras, la valoración simbólica que se resignifica en el consumismo denota una transformación de la relación del consumo respecto a la vida, nos enfrentamos a una nueva fórmula esencial en el plano simbólico: consumo ergo sum.

Interesa señalar también que los contextos urbanos son escenario de otra problemática de gran importancia y tiene que ver con la exclusión social. Para referirnos a este problema adherimos la propuesta de Pérez Sáinz, quien plantea la exclusión social como un proceso extremo de desempoderamiento que deja a población fuera de las dinámicas básicas de participación en la sociedad.

Para este autor, la exclusión primaria se desarrolla en los mercados básicos, campo de poder donde se dan las condiciones de generación, circulación y apropiación del excedente generado por la sociedad. Las desigualdades que se gestan en este campo son de poder, se dan entre clases sociales, individuos y pares categoriales y se generan a través de mecanismos de explotación y acaparamiento de oportunidades. 
La capacidad que tiene la ciudadanía social para neutralizar la exclusión se puede lograr a través del empleo. Fue así que en el momento de modernización nacional se consolidó un núcleo duro de ciudadanía social, mientras que, en la modernización globalizada, el desplazamiento del Estado por el mercado cambió el acceso a la ciudadanía social, así el empleo fue desplazado por el consumo básico de los hogares como mecanismo de ciudadanía (Pérez Sáinz y Mora-Salas, 2006; Mora-Salas y Pérez Sáinz; 2009; Pérez Sáinz, 2014; Pérez Sáinz, 2016). ${ }^{5}$

Con estas premisas analíticas, el estudio se direccionó a explicar la relación entre exclusión social y consumismo, en un contexto urbano afectado por múltiples formas de violencia, poniendo el foco de atención en el segmento de población joven (18 a 29 años).

Figura 1

RELACIÓN ENTRE JÓVENES, EXCLUSIÓN SOCIAL Y CONSUMISMO

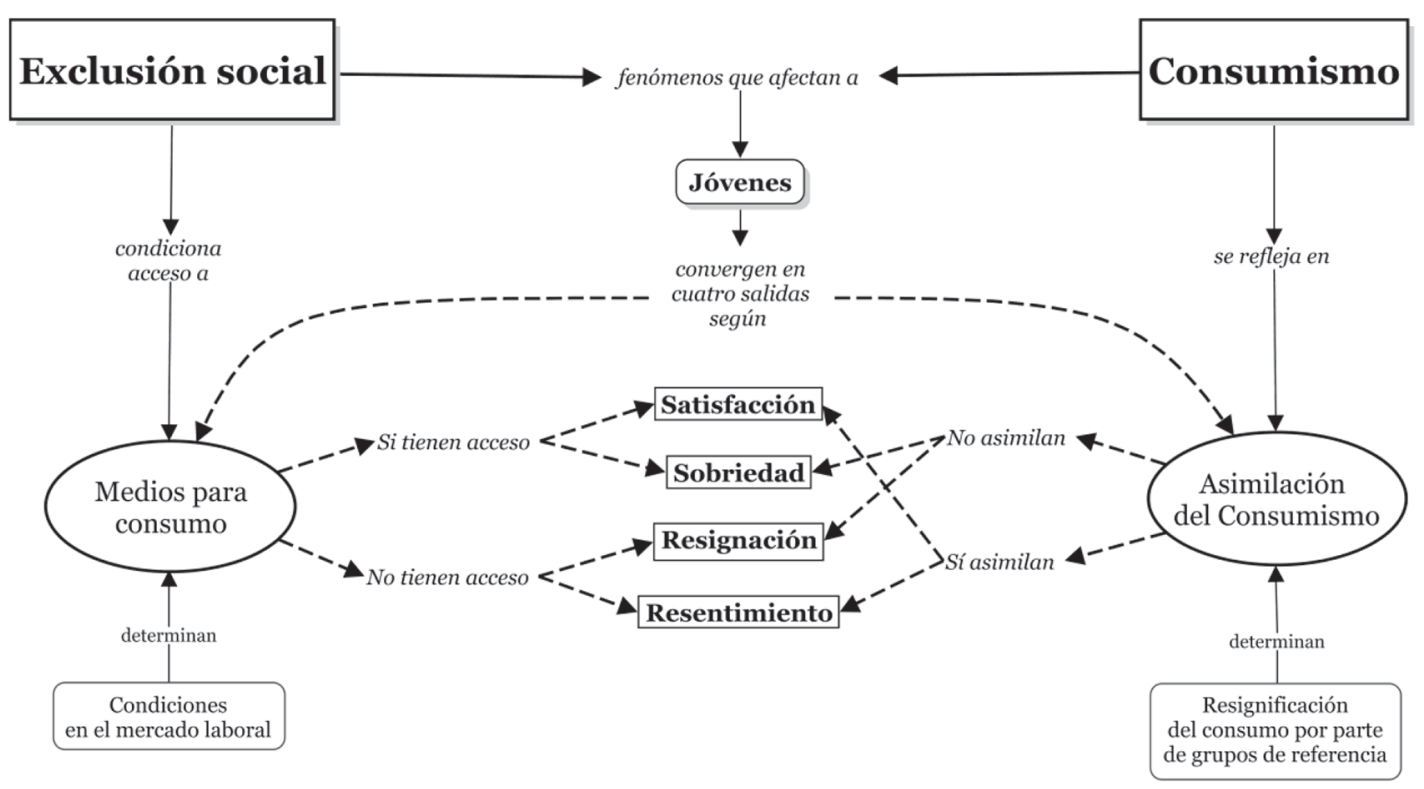

Fuente: Elaboración propia.

La Figura 1 expone la articulación de los dos ejes analíticos de interés, a la luz de la discusión presentada líneas atrás sobre el consumismo y la exclusión social. Empezando por el último fenómeno, existen dinámicas de desempoderamiento en el mercado laboral donde se genera la exclusión, esto afecta a las personas jóvenes en la medida que el acceso a este mercado básico permite la obtención de recursos económicos para satisfacer las pautas de consumo. Mientras que, en el primer fenómeno, el consumismo, la centralidad que tomó el mercado en el proceso globalizador posicionó 
una cultura de consumo donde el valor simbólico de los bienes se coloca como una expresión de estatus y pertenencia social. El consumo como repertorio de legitimación, genera distinción y legitima las desigualdades de fondo al mismo tiempo que amplios sectores de la población asumen estas nuevas pautas culturales, ancladas en el consumo, como medio para generar identidad y pertenencia.

En medio de estas problemáticas se encuentran las personas jóvenes, quienes deben enfrentarse a las vicisitudes para incorporarse a los mercados laborales al tiempo que compiten por garantizar la inclusión simbólica que ofrece el consumismo en los entornos urbanos. ${ }^{6}$ Así, la convergencia de estos problemas se puede plantear en términos de una caracterización de tipos, se tiene así se cuatro salidas distintas. ${ }^{7}$

En primer lugar, en la salida de la satisfacción convergen aquellas personas jóvenes que tienen condiciones favorables en términos de inclusión social, es decir, las condiciones de inclusión le permiten satisfacer necesidades de consumo, es decir, estarían alcanzando las aspiraciones de consumismo que garantizan identidad y pertenencia social.

El segundo escenario es el caracterizado por la sobriedad. Cuando las personas jóvenes tienen poca asimilación del consumismo y al mismo tiempo se encuentra en niveles de inclusión favorable. No se da una aspiración a bienes de consumo como el aspecto más importante para generar identidad a través del consumo; es decir, la identidad y la pertenencia social, a pesar de contar con condiciones favorables en términos de medios de acceso, no se centra en la resignificación del consumo, sino a través de otro tipo de estrategias.

La tercera salida es la resignación, la cual acaece cuando hay niveles altos de exclusión social y la misma situación baja las expectativas para acceder la identidad y pertenencia a través del consumismo. En este caso, la imposibilidad material de acceder al consumo que tiene valor simbólico, este tipo de joven desistiría de visualizar el consumismo como generador de identidad y pertenencia.

Por último, está la salida del resentimiento, el cual acontece cuando la asimilación del consumismo es alta, es decir, la identidad y el sentido de pertenencia social en estos jóvenes está centrado fundamentalmente en las dinámicas consumistas, pero no poseen medios para poder satisfacer esas condiciones por el nivel de exclusión social al que se enfrentan. Se está frente a una situación donde se puede experimentar frustración ante esta incompatibilidad que les deja fuera del reconocimiento social que se gesta en el consumismo, frente a este tipo de jóvenes se plantean las posibilidades de transgresión como una de las salidas.

\section{Contexto de emergencia del consumismo}

La literatura reciente señala que los territorios urbanos en Centroamérica fungen como espacios de articulación de las problemáticas de exclusión social y consumismo. Incluso, las carencias económicas de este tipo de contextos ofrecen oportunidades, 
a través de la transgresión delictiva, para solventar distintas necesidades de la población joven, como la inclusión simbólica que ofrece el consumismo (Beirute-Brealey; Calderón-Umaña; Pérez Sáinz, 2014).

El escenario de surgimiento del consumismo en Centroamérica está marcado por condicionantes históricas que se desenvuelven a finales del siglo anterior: la apertura comercial y la liberalización económica con su impacto en el sector financiero.

El primer fenómeno, la apertura comercial, tiene un antecedente que marca un hito y es el Mercado Común Centroamericano (MCC). Ese esfuerzo integracionista, se acompañó del crecimiento de sectores los económicos de la industria y los servicios, al tiempo que aumentó la cantidad de mercancías disponibles y su circulación. Nuevos bienes de consumo fueron accesibles para los estratos privilegiados que podían acceder a ese consumo suntuario, ofrecido cada vez más a la creciente la población urbana que empezaba a consolidar grupos de clase media (Torres-Rivas).

En ese momento, el simple acceso a esos bienes denotaba un consumo de tipo suntuario, asociado al prestigio y el estatus social. El acceso privilegiado a esos bienes alimentó un imaginario de bienestar en sectores que experimentaron movilidad social. Viales Hurtado señala que en el periodo 1850-1950 en América Central los patrones burgueses de consumo se relacionan con la "modernidad", "en esta época es muy importante la relación entre la intensificación del consumo y la representación, el imaginario, de las clases medias en América Latina, fomentado por el Estado" (VialesHurtado 32).

El crecimiento demográfico y la migración interna en la década del ochenta produjeron cambios. Facilitaron el anclaje urbano del consumo, configurándose una dinámica de socialización alrededor de este, además, se incorporaron nuevos códigos y símbolos que tomaron valor en los procesos de identidad, particularmente en segmentos de población joven. ${ }^{8}$ La acotación territorial viene acompañada de cambios en el paisaje urbano. La aparición de grandes cadenas de supermercados, centros comerciales, centros de entretenimiento, y una serie de "nuevos objetos urbanos", aportaron a esa complejización del consumo, ubicándolo como una nueva dinámica de socialización (Calderón-Umaña; Segura). ${ }^{9}$

Así las cosas, en la modernización globalizada, el acceso a los bienes es necesario, pero no suficiente para obtener prestigio o estatus. Se requiere el manejo e interpretación de una serie de códigos alrededor del consumo para lograr esos fines. Esto fue otorgando un valor simbólico a los bienes, cuya utilidad se da en los procesos de generación de identidad y pertenencia a grupos sociales. ${ }^{10}$

La integración financiera regional, segundo fenómeno de interés, empezó a despuntar algunos rasgos propios en el momento de modernización nacional, ${ }^{11}$ pero es en el momento de modernización globalizada que los vínculos de las elites del sector financiero costarricense logran impulsar una agenda de liberalización, para 
preparar las condiciones de consolidación de grupos financieros centroamericanos y, posteriormente, conexiones con grupos globales.

Estos cambios se cristalizan en Costa Rica en el período 1984-1995. Se identifican tres puntos críticos que favorecen a la liberalización del sector financiero: el primero, en 1984 cuando se da espacio a la banca privada través de un modelo bancario mixto, es decir, una liberalización parcial; segundo, en 1992 se autoriza la intermediación financiera en moneda extranjera, permitiendo a la banca privada financiar a los sectores de más productividad y menor riesgo; tercero, en 1995 la política cambiaria se deja al "libre juego de la oferta y la demanda de divisas"; la llegada de los megabancos en el 2005 termina de sellar este proceso, corolario de lo anterior, se reconfiguraron los grupos de poder que formaron parte del proceso y se ubican ahora como ganadores del ajuste (León Araya; Zúñiga-Ramírez).

Robles Rivera señala la importancia que tiene la apertura comercial en la reconfiguración de los grupos de poder en Costa Rica y El Salvador. La remodelación de la plataforma normativa del comercio, para acoplarse a la internacionalización de los mercados comerciales permitió:

Construir una plataforma jurídica-comercial de expansión amplia para los intereses de los grupos de poder regionales y de las CTM [Corporaciones Transnacionales Metropolitanas]. Fronteras "libres", exoneraciones comerciales, trato nacional y acceso a mercados ha permitido que los grupos de poder regionales amplien y consoliden sus dominios dentro y fuera de la región (Robles Rivera 114).

La extensa oferta de mercancías que circula con la apertura, se acopla a la medida con la nueva oferta de productos financieros que se consolida con la liberalización. Desde los años noventa hay una reducción relativa de recursos dirigidos a sectores agropecuarios, favoreciendo los recursos destinados a la industria y los servicios. Así, "el notable aumento del crédito bancario para el consumo y para vivienda... duplicó su participación desde menos de $25 \%$ en 1995 de la cartera total a más de 50 \% en 2014" (Villamichel-Morales 16-17).

Recientemente el incremento de oferta de préstamos para vehículos o equipo tecnológico, así como la explosión de las tarjetas de crédito y otros servicios propios de las nuevas entidades bancarias, han engrosado los servicios financieros disponibles. ${ }^{12}$ Surgen también alternativas para sectores menos favorecidos económicamente, por ejemplo, se da el "efecto China" (importación de bienes a muy bajo costo); mercados de segunda mano; el contrabando de artículos que imitan marcas reconocidas y el acceso cadenas internacionales de supermercados, todos estos destinados a sectores con menores ingresos económicos y/o sin acceso a los mecanismos formales. La transgresión también aparece en la ecuación como un mecanismo de acceso en contextos signados por la exclusión (Calderón-Umaña; Pérez Sáinz, 2014). 
La simbiosis entre estos fenómenos -apertura comercial y liberalización financiera- abre espacio para la consolidación del consumismo en la región. En términos de la nueva conformación del paisaje urbano y de la segregación urbana, el distrito de Los Guido también se vio afectado por dichos fenómenos desde su momento fundacional, ya que se ubica como una zona urbana periférica que absorbe contingentes de población y concentra un importante segmento de la población económicamente activa que vende su fuerza de trabajo a estas nuevas actividades comerciales (Mora-Salas y Solano Castro).

Asimismo, estos aspectos evidencian que la emergencia del consumismo tiene estrecha relación con el mundo urbano, pues es el escenario territorial en el que se ancla materialmente este fenómeno, además, converge con las condiciones de exclusión social y los efectos de la segregación urbana, como ya hemos señalado. La generación de identidad y la pertenencia social que reviste el consumismo, así como la compleja dinámica que conlleva, le han otorgado un papel central en este tipo de territorio; lo fundamental es caracterizar estos aspectos.

\section{Condiciones de origen de Los Guido de Desamparados}

En Costa Rica, durante los años ochenta, se dan movilizaciones intra e interurbanas y un crecimiento demográfico importante. En este periodo las reconfiguraciones de las relaciones productivas del país fueron atravesadas por el auge y fortalecimiento del neoliberalismo. Dichas condiciones estructurales dieron paso a una relocalización espacial de grupos de menores ingresos a nuevos asentamientos urbanos. La parte precaria de esta segregación urbana se concentró en mayor medida en algunos distritos del sur de San José (Mora-Salas y Solano Castro).

Este proceso de cambio en las áreas metropolitanas se vinculó estrechamente con el surgimiento de distintos frentes de vivienda que se mantuvieron tensiones políticas luchando por el acceso a soluciones habitacionales. Paulatinamente las intervenciones en esos espacios fueron generando focos poblacionales densos, caracterizados mayormente por la presencia de pobreza urbana. Se fue agotando la disponibilidad de espacios centrales, ampliándose el rango de intervención de los espacios hacia otras áreas periurbanas del Área Metropolitana de San José (Mora-Salas y Solano Castro).

En este contexto que, en 1986, se da una toma de tierra en el distrito de Patarrá, en una propiedad perteneciente a la familia Guido Von Schroeter (de ahí el origen del nombre), que da origen al asentamiento de Los Guido (Mata). En ese momento, se caracterizó por la presencia de construcciones precarias, falta de acceso a los servicios básicos de agua y electricidad y desorganización espacial del territorio. El crecimiento demográfico y territorial fue paulatinamente consolidando el asentamiento como una comunidad urbana de gran volumen; en el 2003 se nombra décimo tercer distrito del cantón de Desamparados (Presidente de la República et al.). 
A pesar del impacto de la segregación urbana en Los Guido, el distrito tiene en la actualidad una conformación heterogénea. Existen sectores con viviendas en condiciones regulares o buenas, aunque otros sectores conservan aún las características del momento fundacional. Sin embargo, un elemento común que se instauró en este territorio tiene que ver con una problemática que ha venido carcomiendo la convivencia social en toda su extensión: la presencia de micromercados de droga. ${ }^{13}$

Este problema tiene raíz en el control territorial que se ha desarrollado desde el momento fundacional. Se han caracterizado tres etapas del mismo: la primera, se extiende desde el origen del asentamiento hasta el año 2005 y estuvo asociada al dominio de cuatro pandillas ${ }^{14}$ sobre sectores específicos de Los Guido, este tipo de control se desvanece hacia el año 2009. En ese momento inicia la segunda etapa, que se extiende hasta el 2015, y se caracteriza por desenvolvimiento de la figura del búnker ${ }^{15}$ como elemento central para la venta de droga, propiciando a su vez otras situaciones delictivas y violentas. ${ }^{16}$ La tercera etapa se desarrolla del 2015 en adelante y consiste en una adaptación logística en el control de los micromercados de droga, incorporando la venta de drogas desde "puntos móviles" que se desplazan por todas las zonas del territorio. Este control territorial tiene consecuencias en términos de vulnerabilidad para las personas jóvenes del distrito, y a la población general, tanto en posibilidades de victimización, como en oportunidades de transgresión (Hernández Ulloa y Pérez Sáinz, 2014).

Esta visión panorámica de Los Guido da cuenta del entramado social que se teje en este tipo de territorios urbanos, particularmente lo que acontece con las personas jóvenes que empiezan a ocupar un lugar más visible en la sociedad y que sin duda son un agente clave en proceso de transformación social, pero al mismo tiempo deben afrontar este tipo de retos en la inmediatez del territorio que habitan.

\section{Apuntes cuantitativos para el estudio del consumismo y la exclusión social}

Como ya se señaló, el objetivo de la investigación fue analizar críticamente cómo se expresan las relaciones entre consumismo y exclusión social y de qué manera afecta a las personas jóvenes de 18 a 29 años del distrito de Los Guido, en el cantón de Desamparados. Para tal fin, se desarrolló una metodología en dos fases. Este texto abordará únicamente lo referido a la primera fase, de carácter cuantitativo, para profundizar sobre hallazgos centrales y presentar varias precisiones metodológicas. ${ }^{17}$ Esta etapa consistió en la aplicación de una encuesta a personas jóvenes de Los Guido, se presentan a continuación dos ejercicios que se realizaron para analizar los resultados de la misma. ${ }^{18}$

Para la encuesta se elaboró un diseño muestral bietápico estratificado cuya población de estudio fueron jóvenes entre 18 y 29 años de edad residentes del distrito 
de Los Guido de Desamparados. Se obtuvieron 467 casos (de jóvenes) con un nivel de confianza del $95 \%$ y un error de muestreo de $+/-4.34 \%$ y representatividad de los resultados según sexo y grupo etario. ${ }^{19}$

El primer ejercicio realizado fue la construcción de una escala de exclusión/ inclusión social y una escala de asimilación del consumismo. La primera escala consiste en la operacionalización del fenómeno de la exclusión en sus dos dimensiones. Esta construcción tiene un anclaje teórico-metodológico en la propuesta desarrollada ampliamente por Pérez Sáinz y Mora-Salas (Pérez Sáinz y Mora-Salas 2006; Mora-Salas 2012; Pérez Sáinz 2012, 2015).

Primero, se elaboró una Escala de inclusión/exclusión social (EIES) trabajando con los datos de jóvenes en diferentes condiciones de actividad: asalariados/as (incluye trabajadores asalariados/as, trabajadores no remunerados/as), desempleados, trabajadores por cuenta propia e inactivos/as (incluye los que estudian, hacen solo tareas en el hogar y realizan otras actividades). Esta escala se compone a su vez de dos subescalas: la de inserción laboral (EIL) y la de ciudadanía social (ECS).

La EIL se dividió en dos, según la condición de actividad de la persona joven. Por un lado, la Escala de Precariedad Salarial (EPS) que se aplicó a jóvenes asalariados e incorpora también a los desempleados. Por otro lado, la Escala de Acumulación (EA) se aplicó a jóvenes que se dedican a una actividad o negocio propio. Al grupo de jóvenes inactivos no se les aplicó la EIL, porque no forman parte de la Población Económicamente Activa (PEA), es decir, no están en el mercado de trabajo. ${ }^{20}$ La EPS mide las condiciones de explotación y acaparamiento de oportunidades de la fuerza de trabajo juvenil mientras que la EA se aproxima a las condiciones de acceso a educación y seguridad social de las personas jóvenes y sus hogares. La ECS se construyó a partir de variables proxy, pues al plantear a la persona joven como unidad de análisis, se obtuvo poca información sobre otros miembros del hogar. Esta escala se elaboró a partir del acceso a seguridad social y los niveles de instrucción. Finalmente, el promedio simple entre la ECS y la EIL dejan como resultado final la EIES. ${ }^{21}$

La otra escala elaborada es una propuesta original para medir la asimilación del consumismo. Es decir, el nivel de incorporación por parte de los jóvenes acerca de la necesidad de artículos -referenciados como icónicos del consumismo-, cuyo valor simbólico es altamente apreciado en los procesos de identificación y pertenencia social. A cada artículo considerado, la persona joven le atribuyó un nivel de necesidad, que luego se entiende como intensidad de asimilación del consumismo a partir de criterios de selección que los informantes tomarían en cuenta si tuviese la oportunidad de adquirir el ítem. ${ }^{22}$

Para garantizar la solidez de estas escalas, se realizaron sendos análisis de confiabilidad, tanto para la EIL como para la EAC. Se acudió a la prueba de Alfa de Cronbach para ese fin. Los resultados están entre el valor 0 , que significa que los ítems de la escala no guardan ninguna relación, y el valor 1, que significa que el mayor 
nivel de relación entre los ítems. La literatura especializada señala que un Alfa de Cronbach superior a 0.8 significa que es un índice confiable, sin embargo, también se ha señalado que el uso de este indicador debe tener en cuenta elementos analíticos atinentes al problema de investigación, es decir, este indicador señala la confiabilidad del índice que se pretende elaborar, pero no debe determinar la construcción del mismo (Mora-Salas, 2012).

El ejercicio de confiabilidad realizado con todos los estándares laborales de la subescala de precariedad laboral arrojó, en el primer análisis realizado tomando en cuenta los 10 elementos, un Alfa de 0.786, este valor se puede maximizar suprimiendo de la escala el estándar de "jornada laboral", obteniendo un Alfa de 0.815 en la escala con nueve ítems. Con respecto al mismo ejercicio de confiabilidad para la escala de asimilación del consumismo, se obtuvo el máximo valor posible para ese conjunto de variables con el primer procesamiento, un Alfa de 0.716, y el índice no puede ser mejorado eliminando algún ítem. Tomando en cuenta lo señalado anteriormente, se considera que la escala es suficientemente confiable para ser tomada en cuenta. ${ }^{23}$

La información obtenida en las escalas permitió ubicar los casos en un plano cartesiano, segundo ejercicio realizado. En el eje x se ubica la escala de asimilación del consumismo y en el eje y la escala de exclusión/inclusión; ambos ejes se mueven entre valores 0 y 10 . Sobre este plano se ubican los conglomerados, de acuerdo a los respectivos centroides; cada conglomerado es incorporado en una "burbuja" cuyo tamaño representa el porcentaje de casos que contiene cada uno, obteniendo así una tipología de jóvenes. Esto se realizó a partir del análisis de conglomerados bietápicos (Two-step clusters), técnica que permite generar agrupaciones de casos de acuerdo a la dispersión de los datos en dos ejes.

Este ejercicio se considera pertinente por la facilidad para identificar asociaciones entre las dimensiones vertidas en las dos escalas expuestas párrafos atrás. A pesar de que existe un método de fijación de conglomerados a priori que podría facilitar la distribución de los casos en los cuatro tipos planteados desde la hipótesis de trabajo, se decidió optar por la ruta de $k$-means, que define una cantidad centroides ajustándose en la mayor medida posible a la estructura del conjunto de datos analizados.

\section{Satisfacción, sobriedad, resignación y resentimiento: Salidas de las personas jóvenes frente a la exclusión social y el consumismo}

El Figura 2 muestra los resultados obtenidos del ejercicio de análisis bietápico de conglomerados. En primera instancia, la lectura sobre estos resultados se realiza a la luz de la precisión metodológica señalada sobre la aplicación de la técnica del análisis de conglomerados. Es decir, la cantidad resultantes de conglomerados no corresponden necesariamente a las hipótesis que se plantearon como resultado de la 
convergencia de los dos fenómenos en estudio. Al contrario, con el fin de contrastar la hipótesis, se decidió no definir a priori la cantidad de conglomerados esperados. El primer hallazgo que se reconoce es la heterogeneidad de juventudes presentes en el territorio, es decir, jóvenes que comparten un mismo contexto se encuentran en posiciones diferenciadas con respecto a los fenómenos de interés. Las formas en que confluyen la exclusión social y consumismo permiten identificar siete tipos de jóvenes distribuidos en el plano cartesiano.

Así las cosas, se puede realizar una lectura de la Figura 2 desde dos grupos de conglomerados. Por un lado, se identifican los cuatro tipos de jóvenes propuestos en la hipótesis de trabajo, por otro lado, tres tipos más que emergieron del procesamiento de los datos.

Del segundo grupo destaca que concentra al $55.5 \%$ del universo de estudio, sin embargo, las posiciones de estos tres conglomerados los desplaza del foco de atención de la hipótesis de trabajo. A pesar de que comprende poco más de la mitad de la población, nos enfrentamos a una limitación analítica, en términos de que las posiciones intermedias no permiten contrastar las características esenciales de los fenómenos en estudio, a pesar de que existe cierta variabilidad entre estos conglomerados. El primer grupo concentra el resto de población y está conformado por los cuatro conglomerados que se ubican en las posiciones extremas del plano. El análisis se acotará a estos cuatro tipos, pues contienen las situaciones límite en las relaciones entre exclusión social y consumismo. 
Figura 2

TIPO DE JOVEN SEGÚN NIVELES DE EXCLUSIÓN/INCLUSIÓN SOCIAL

Y ASIMILACIÓN DEL CONSUMISMO

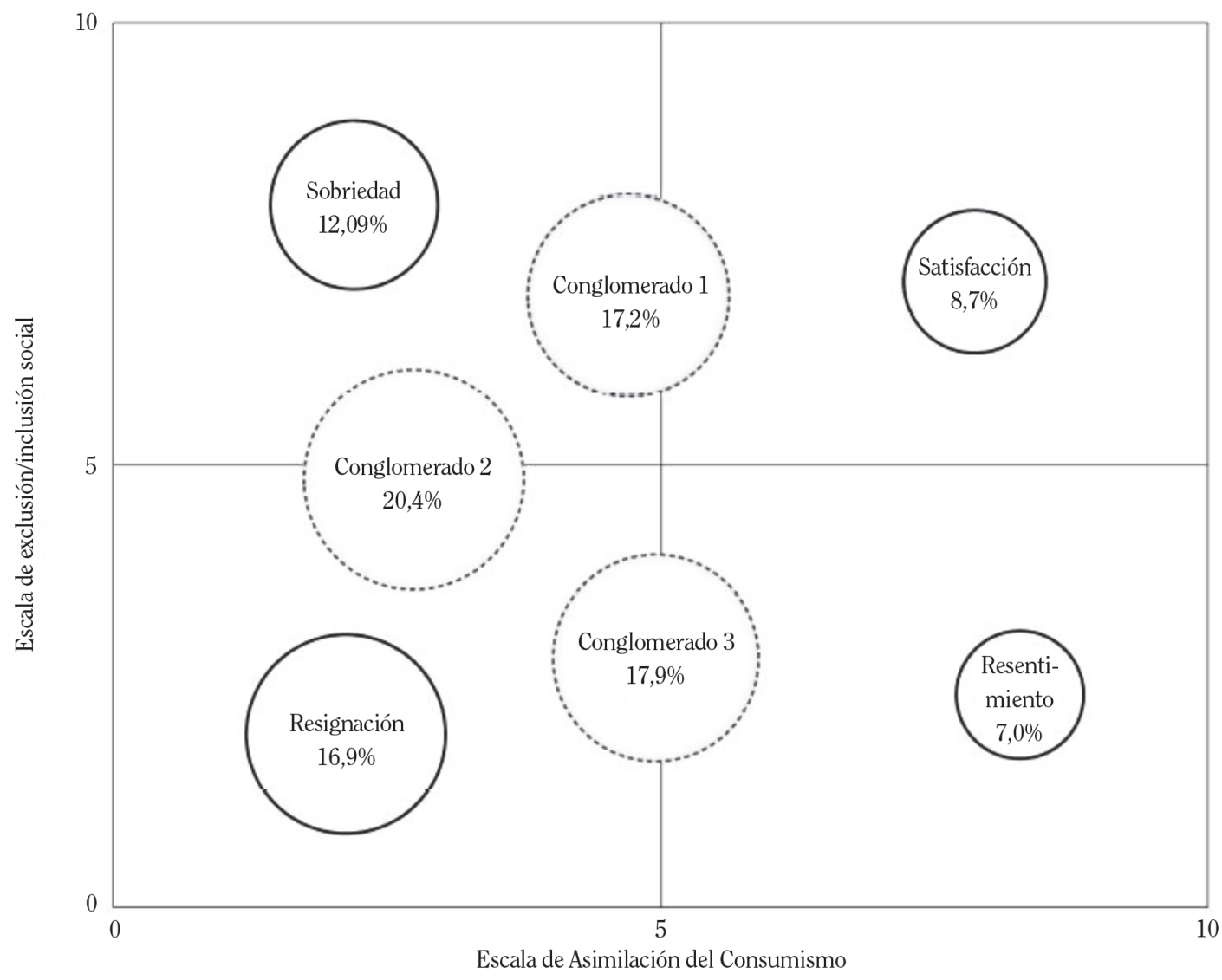

Fuente: Elaboración propia.

Antes de avanzar en el análisis, merece la pena detallar tres aclaraciones metodológicas. La primera es que, tal y como lo reflejan la Figura 2 y la Tabla 4 (Anexo metodológico), el análisis de conglomerados produce como resultado una distribución de la totalidad de casos del universo de estudio a lo largo del plano cartesiano, por lo tanto, las agrupaciones de casos representadas en las burbujas "tipos" están determinadas por la aplicación de la técnica y no han sido determinadas de manera apriorística, además, las escalas empleadas para realizar el ejercicio fueron sometidas a la validación del Alfa de Cronbach, aspecto que se puede consultar con detalle en el anexo metodológico. 
En segundo lugar, la decisión de dejar fuera del análisis de perfiles a los conglomerados 1, 2 y 3 resulta del interés de enfocar la atención en aquellos que los tipos ideales planteados en el marco analítico. Lejos de ser una adecuación de datos al modelo, este ejercicio pretende detallar a profundidad las características de los tipos de jóvenes que representan con claridad la conjugación de exclusión social y asimilación del consumismo, debido a que son los temas que se quieren explorar, las cuatro salidas a la convergencia de estos fenómenos.

En tercer lugar, los demás tipos no son profundizados, pero se sabe de su existencia y quedan abiertas las interrogantes sobre el comportamiento de las variables que explican las posiciones intermedias tanto en la escala de exclusión social como la de consumismo. Si se quiere, se puede plantear que estos conglomerados son una quinta salida a la convergencia de los fenómenos de estudio. Aunque, se asume el vacío existente al dejarlos por fuera en la revisión de perfiles con el afán de priorizar a los tipos relacionados estrechamente con las hipótesis de trabajo y el objeto de estudio, es decir, a aquellos que se ubican en los extremos de ambas escalas, con las combinaciones ya formuladas.

Junto a los datos presentados en la Figura 2, se analizaron perfiles sociodemográficos que presentan características con diferencias estadísticamente significativas entre los cuatro conglomerados. La Tabla 1 recupera la posición de cada grupo según los niveles en la escala, el peso del grupo en el universo de estudio y las variables destacadas en los perfiles. 


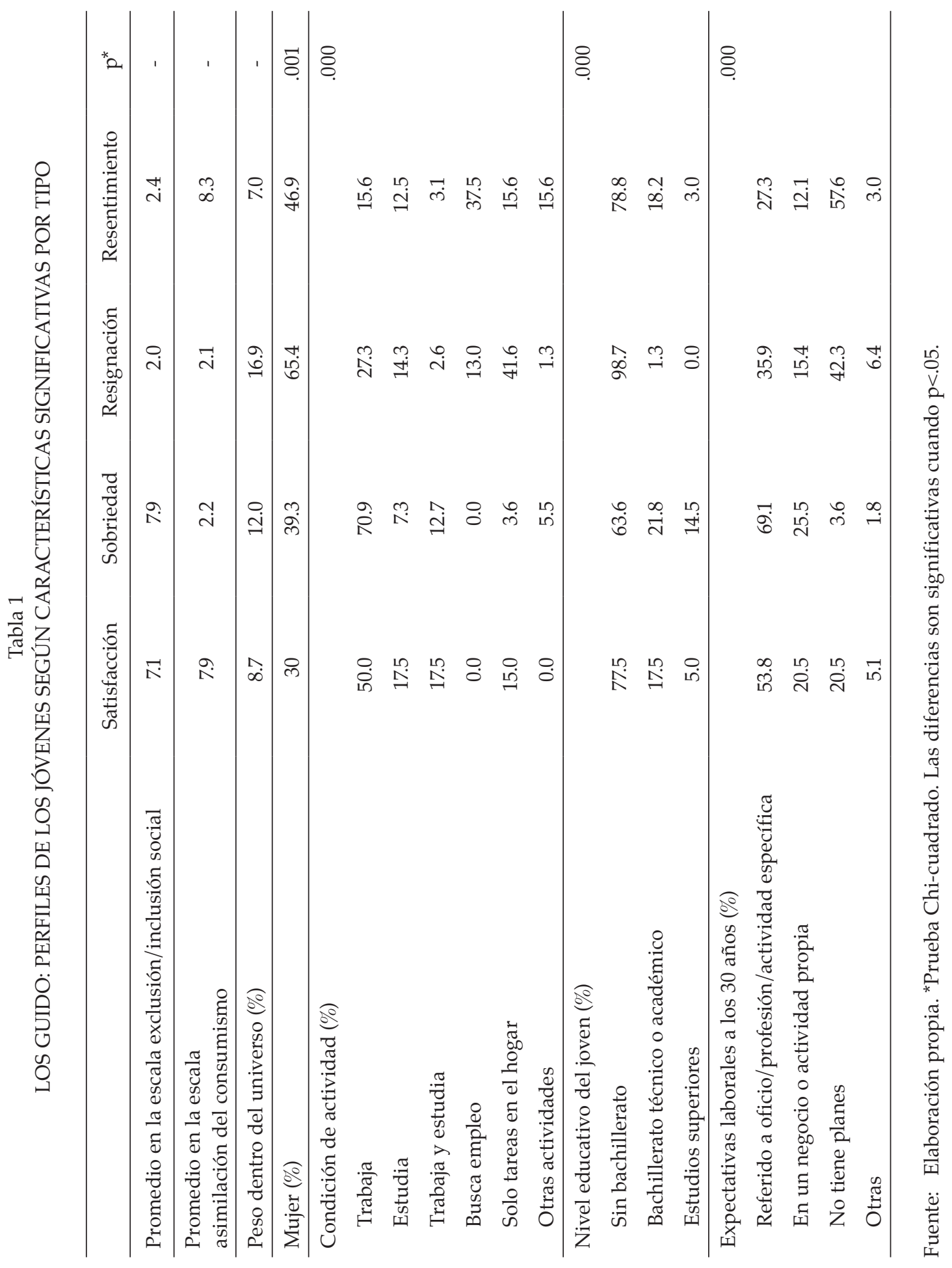


Los perfiles que se reflejan en la Tabla 1 se abordarán por cada uno de los tipos. Sobre jóvenes de tipo satisfechos, lo primero que resalta es que estamos frente a un tipo confirmado mayoritariamente por hombres jóvenes insertos en el mercado laboral, principalmente a través del trabajo. Esto significa que existen fuentes de ingresos que les permite satisfacer sus necesidades de consumo. A pesar de que este grupo concentra la mayor proporción de jóvenes que han cursado estudios superiores, en todos los grupos predomina la falta de este logro académico con más o menos fuerza. Sin duda alguna, esto supone dificultades en la inserción en el mercado laboral, en el caso de los jóvenes que logran insertarse, la vulnerabilidad a caer en situaciones de empleo precario está latente. ${ }^{24}$

Esto cuestiona el tipo de consumo al que pueden acceder estos jóvenes. Si bien es cierto, en comparación con los demás tipos se encuentran en una condición muy favorable, la situación insuficiente en términos de logro académico -además, generalizada para todos los jóvenes del distrito- cuestiona la sostenibilidad de esa condición, pues en la actualidad la garantía de ubicarse en posiciones de inclusión social está dada por su participación en el mercado laboral.

Con respecto a las expectativas futuras sobre el trabajo, la mayoría de estos jóvenes espera estar trabajando a los 30 años, aquí llama la atención que esperan un futuro laboral bastante determinado. Esa visión destaca por las escasas oportunidades para mantenerse en los estudios e insertarse al mercado laboral que tienen estas personas jóvenes en el territorio, además del riesgo existente de ser víctima de alguna manifestación de violencia. Es decir, estamos frente a un escenario poco motivador para imaginarse un futuro y, aun así, un grupo importante de jóvenes lo hace, apostando a la inserción laboral.

El segundo tipo de joven corresponde a la sobriedad. Para este tipo de jóvenes los bienes de consumo no aparecen como prioritarios, a pesar de disponer de recursos para acceder a estos. El impacto positivo de la inserción laboral también favorece a este grupo, se puede notar que la no participación en el estudio o el trabajo es reducida. Es decir, estamos frente a jóvenes que se encuentran activamente desarrollando su proyecto de vida de manera propositiva en el trabajo o en el estudio, es el grupo con mejores condiciones de inclusión y, al igual que grupo anterior, este tipo de joven visualiza el futuro en términos laborales y, principalmente, en términos educativos porque han logrado un importante proceso de escolarización.

En términos de la visión sobre el futuro, se proyectan con una tendencia más orientada al logro educativo que en el caso anterior, incluso, priorizando ese tipo de logro sobre la satisfacción de consumo. Esto se devela en la fuerte proyección hacia el logro profesional y una relativa ausencia de incertidumbre en este tipo de jóvenes. Es claro que esto está posibilitado por el nivel de inclusión social favorable que tiene estos jóvenes, el promedio más alto en la escala de inclusión/exclusión social. 
El tercer tipo de jóvenes corresponde a un conglomerado que agrupa las condiciones de exclusión social más desfavorables en el distrito. La valoración sobre el consumismo que tiene este grupo también es la más baja del distrito, son quienes perciben los bienes de consumo como totalmente innecesarios. Esta combinación de posiciones se puede interpretar como resignación ante la imposibilidad de aspirar al consumismo como salida, a sabiendas de que su situación desfavorable no les permitirá acceder a estos. La composición mayoritaria es de mujeres confinadas al espacio doméstico, sabiendo que detrás de esta figura se oculta el trabajo doméstico no remunerado. La condición de exclusión del mercado laboral, les ubica como jóvenes que no aspiran al consumismo como dinámica que les genere identidad o pertenencia.

Casi la totalidad de jóvenes en este grupo no han obtenido el bachillerato; se trata de uno de los aspectos que los hace tender a la exclusión social, además de ser un obstáculo para salir de esa condición. La presencia dominante de mujeres en el trabajo doméstico no remunerado anuncia que estamos frente a una situación de enclaustramiento con tintes de dominación masculina que limita las posibilidades de estas jóvenes de tener una juventud proyectada hacia el futuro, la caracterización general de la comunidad mostró que el abandono escolar en el distrito está marcado por responsabilidades económicas familiares en el caso de los hombres y responsabilidades de cuido en el caso de las mujeres. ${ }^{25}$

El último grupo, es la situación opuesta por el lado del consumismo. Se encuentran en una situación perversa, cargada de frustración, tanto que no se tienen condicione favorables de inclusión al tiempo que se asimila el consumismo como aspecto importante, frente a esto, se da una posible actitud de resentimiento, en tanto que existe una visión del consumo como aspecto necesario en su vida y la condición desfavorable le limitaría el acceso a esta situación. En el perfil destaca que la presencia de mujeres también es alta y las brechas en las condiciones de actividad con los otros grupos son importantes. Estamos frente a al tipo de joven que experimenta la exclusión laboral con intensidad. El acceso a estudios superiores es altamente deficiente, se arrastran desventajas en el plano laboral y educativo al mismo tiempo, similar que el grupo anterior, con la diferencia que el consumismo se ubica en un plano importante de las vidas de estos jóvenes.

En el plano de expectativas a futuro, este tipo de jóvenes contrastan con los datos de los primeros dos tipos. Sostienen la tendencia de expectativas orientadas al mundo laboral, sin embargo, las expectativas laborales aparecen como una figura que se desvanece. ${ }^{26}$ La minoría visualiza una salida laboral o educativa para lograr mejorar la condición de exclusión social, y por ende acceder con mayor facilidad a las dinámicas de consumo que tienen un papel importante en sus vidas. 


\section{Conclusiones}

Los contextos urbanos signados por la violencia están compuestos por una amplia diversidad de formas de juventud, tal y como se evidenció en el distrito de Los Guido. La agrupación de personas jóvenes por tipos señaló que poco más de la mitad se ubica en condiciones intermedias frente a la exclusión social y el consumismo, sobre este grupo no se profundizó el análisis, sino que se puso la mirada sobre la otra mitad de jóvenes, quienes se mueven entre cuatro dinámicas que se ubican en los extremos de las dimensiones de estudio. La conformación de los hogares no sugiere diferencias entre los tipos, sin embargo, cuatro características sociodemográficas que marcaron diferencias significativas: el sexo, la condición de actividad, el nivel educativo y las expectativas laborales a futuro.

Las primeras dos variables se entrelazan. La evidencia empírica apunta a la presencia de desigualdades de género en distintas dimensiones. El tipo de joven en resignación está representado principalmente por mujeres que se dedican a tareas en el hogar, es decir, trabajo doméstico no remunerado. Este es el grupo que se ubica en la peor posición de ambas escalas. Estas jóvenes se ven afectadas por la reclusión en la esfera doméstica afecta, las responsabilidades en sus hogares inciden en la interrupción de sus estudios dificultando su incorporación al mercado laboral. Enfrentar esta condición de exclusión lleva a estas jóvenes a posicionarse con resignación y de manera evasiva ante la posibilidad de lograr inclusión simbólica a través del consumismo, pero también les refuerza una visión limitada sobre el futuro, en los pocos casos en la que existe. La falta de espacios de sociabilidad y la constante exposición a la violencia coadyuva a que los horizontes del futuro queden atrapados en ese confinamiento. ${ }^{27}$ Estamos entonces ante una doble consecuencia negativa, se padece la exclusión social y se revela la fragilidad del consumismo como mecanismo de identidad y pertenencia social en el plano simbólico.

El escenario se complementa de manera negativa con la poca presencia de mujeres en los tipos de jóvenes que tienden a la inclusión, quienes poseen mejores condiciones de acceso al mercado laboral y mayor logro educativo. En otras palabras, la fuerza de trabajo del distrito está masculinizada, favoreciendo a que los hombres en general tengan mejores condiciones de inclusión social. Esto también incide, en lo particular, en que jóvenes que tienen condiciones de inclusión favorables y altos niveles educativos (el tipo sobrio) sobrepongan las opciones de continuidad educativa sobre la satisfacción del consumismo, ya que sus perspectivas sobre el futuro están orientadas al logro educativo/laboral. Aquellos con condiciones favorables de inclusión, pero menos trayectoria educativa, logran satisfacer el consumismo, sin embargo, se enfrentan a la vulnerabilidad laboral por sus bajas credenciales educativas.

Las expectativas laborales a los 30 años revelaron dos tendencias marcadas. Del lado de los tipos que tienden a la inclusión social (satisfechos y sobrios), las expectativas 
se depositan en escenarios específicos, es decir, estos jóvenes se imaginan trabajando en oficios/profesiones/actividades específicas. Esto es congruente con su actual situación de inserción en el mercado laboral -además con bajo impacto de la precariedad laboralo inserción educativa, y la concentración de jóvenes que han logrado acceder a la educación superior. Del lado de los que tiende a la exclusión social (resignados y resentidos), la carencia de planes laborales es lo que domina las expectativas sobre el trabajo. No se debe descartar el porcentaje de jóvenes que aspiran a escenarios específicos, pero se asume que en los casos en los que existe este tipo de proyección, la orientación consumista juega un papel importante, especialmente en el caso de los jóvenes resentidos.

Estos perfiles de jóvenes se complementan con algunas hipótesis sobre mecanismos que emplean estas personas jóvenes para acceder al consumismo. En ese sentido jóvenes satisfechos optarían por mecanismos de tipo normativo para lograr su satisfacción del consumismo; jóvenes sobrios acuden a mecanismos evasivos, por su baja asimilación del consumismo, pero si fuera el caso, tiene medios para acceder a mecanismos normativos de igual forma; entre jóvenes resignados predominaría mecanismos evasivos, mientras que jóvenes resentidos se encontrarían en una zona de vulnerabilidad a la trasgresión, como alternativa para satisfacer el consumismo dado las condiciones de exclusión social que enfrenta. ${ }^{28}$ Es decir, la diversidad de juventudes también presenta acciones diferenciadas frente a sus condiciones de exclusión social y asimilación del consumismo. Esto es una ruta de investigación que se abre para profundizar sobre las estrategias de jóvenes de estos contextos para afrontar estas problemáticas.

Por último, la investigación permite concluir que el consumismo, a pesar de ser una dinámica que se transformó en generadora de identidad por el impacto de la globalización, está marcada por su carácter individualista y competitivo que supone que la adquisición de bienes va a garantizar inclusión. Lejos de esto, lo que se da es una legitimación de las desigualdades que acontecen previo al consumo, mismas que se relativizan frente a la inclusión simbólica. En pocas palabras, el consumismo no resuelve los problemas derivados de la exclusión social, más bien refuerza y legitima las desigualdades que la generan y torna, a quienes participan de esta dinámica, vulnerables. 


\section{ANEXO METODOLÓGICO}

\section{Diseño muestral}

Población de estudio: Jóvenes de 18 a 29 años residentes en el distrito de Los Guido del cantón de Desamparados.

Diseño General: Diseño estratificado (seis zonas geográficas de Los Guido validadas por un líder comunitario ${ }^{29}$ bietápico con selección sistemática de Unidades Primarias de Muestreo (247 segmentos de aproximadamente veinte viviendas), selección total de Unidades Secundarias de Muestreo (viviendas con al menos un residente entre 18 y 29 años), y selección simple al azar de Unidades Terciarias de Muestreo (persona joven en el rango etario señalado).

Marco muestral: Se ha tomado como base el mapa de Los Guido provisto por la municipalidad de Desamparados el cual posteriormente fue actualizado. De esta forma, se ha tenido un marco de áreas sin información específica sobre cada una de ellas, salvo el número total de viviendas dentro de cada área.

Tamaño de muestra efectivo y tasa de respuesta: se consiguió entrevistar a 460 de los 1316 jóvenes que se esperaba entrevistar, para una tasa de no respuesta de $65 \%$. Por error de ubicación, se realizaron 7 entrevistas en segmentos adyacentes a los seleccionados en la muestra. Se tomó la decisión de no perder estas entrevistas e incorporarlas a la muestra cuyo tamaño final ha sido de 467.

\section{Construcción de las escalas}

\section{Escala de Inclusión/Exclusión Social (EIES)}

Para elaborar la EIES se trabajó con los datos de jóvenes en diferentes condiciones de actividad agrupados en cuatro: asalariados/as (incluye trabajadores asalariados/as, trabajadores no remunerados/as), desempleados, trabajadores por cuenta propia e inactivos/as (incluye los que estudian, hacen solo tareas en el hogar y realizan otras actividades). Esta escala se dividió en dos.

\section{Escala de Inserción Laboral (EIL)}

Esta escala permite medir, a partir de la información laboral de los jóvenes, la exclusión primaria en el mercado laboral. La escala tiene valores que van de 0 a 10 , donde máximo nivel de desempoderamiento está marcado por el valor 0 , mientras que el extremo opuesto de la escala se declara con el valor 10. A continuación, se detalla la elaboración de las misma las escalas de Precariedad Salarial (EPS) -incorpora las personas jóvenes asalariadas y desempleadas- y la Escala de Acumulación (EA). 


\section{Escala de Precariedad Salarial}

Es una escala acumulativa compuesta, que utiliza una serie de estándares laborales asociados a la calidad del empleo para captar la precariedad laboral, ${ }^{30}$ asignándole a cada estándar el valor 0 cuando no se cumple y el valor 1 cuando si se cumple, los estándares a considerar son los siguientes: estabilidad laboral; jornada laboral; salario mínimo; ${ }^{31}$ forma de pago; días pagados por enfermedad; vacaciones; aguinaldo; seguro de riesgo de trabajo; horas extra; seguro social.

El máximo nivel de precariedad laboral estaría signado por los jóvenes que están desempleados. Para incorporarlos en la EPS se hace una distinción, aquellos que llevan menos de 12 meses buscando empleo se incorporan a la escala con valor 0.5, mientras los que llevan más 12 meses o más -conocidos como desalentados- tendrían el valor 0 , que es el mínimo de la escala.

Esto deja una escala aditiva compuesta, donde a la cantidad de estándares laborales que posee la persona joven se le suma 1 (el valor 0 ya están asignados, por lo que el valor 1 corresponde al mínimo disponible, que se le asigna a quienes no poseen ningún estándar). Resultando que: $0=$ desempleados durante 12 meses o más; $0.5=$ desempleados por menos de 12 meses; 1 = empleado sin ningún estándar laboral; 2 = un estándar laboral; 3 = dos estándares laborales; 4 = tres estándares laborales; 5 = cuatro estándares laborales; 6 = cinco estándares laborales; 7 = seis estándares laborales; $8=$ siete estándares laborales; 9 = ocho estándares laborales; 10 = nueve estándares laborales.

\section{Escala de Acumulación}

Es una escala acumulativa que toma como base el número de trabajadores remunerados para su negocio o actividad y a partir de ahí se modula con dos criterios más: el tipo de contabilidad que lleva para la actividad y la ubicación dónde se desarrolla. Por un lado, cuando no lleva contabilidad se reduce el valor de la escala a la mitad (se multiplica por .5), cuando lleva contabilidad en un cuaderno se reduce la escala un tercio (se multiplica por 0.75 ) y cuando la contabilidad es llevada por un contable se conserva el valor de la escala (se multiplica por 1). Por otro lado, cuando la actividad se realiza fuera de la vivienda o en la vivienda, pero tiene medidor de electricidad separado, se conserva el valor original (se multiplica la escala por 1), la actividad se realiza en la vivienda y no tiene medidor aparte, se reduce el valor de la escala a la mitad (se multiplica por .5).

Teniendo estás dos subescalas se puede generar la EIL, compuesta por valores de la EPS para los asalariados y los valores de la EA para trabajadores por cuenta propia. A las personas jóvenes de las demás categorías de condición de actividad, fundamentalmente inactivos/as, no se les aplicó valores de esta escala. 


\section{Escala de Ciudadanía Social (ECS)}

Esta escala mide el acceso a ciudadanía social, al ser el joven la unidad de análisis, se obtuvo poca información sobre otros miembros del hogar, esto implicó el uso de variables proxy para las dos subescalas que la componen.

\section{Escala de Seguridad Social (ESS)}

Esta subescala se calcula con la cantidad de personas que dependen del seguro social por cada persona que tiene seguro social (obtenido a través del trabajo), ${ }^{32}$ se le asigna la mitad del valor a los que están asegurados de manera indirecta (multiplica por .5) y se deja con valor 0 aquellos hogares sin asegurados por empleo; el menor valor en la escala significa menos personas aseguradas por hogar.

\section{Escala de Credenciales Educativas (ECE)}

Para esta subescala se asigna un valor a los diferentes niveles educativos de la siguiente forma: 0 = educación especial; 1 = primaria incompleta; 2 = primaria completa; 3 = secundaria académica incompleta; 4 = secundaria técnica incompleta; 5 = bachillerato académico con título; 6 = bachillerato técnico con título; 7 = educación superior.

Esta escala se aplica tanto para el joven como para la persona con mayor nivel del educativo en el hogar, se calcula un promedio simple entre dichos valores para obtener una escala donde el valor mínimo (0) representa el menor credencial educativo y el valor máximo (10) representa el mayor credencial.

Con el cálculo de la ECE y la ESS se calculó un promedio simple para obtener la ECS, seguidamente se obtuvo el promedio simple entre la ECS y la EIL, teniendo como resultado final la EIES. ${ }^{33}$

\section{Escala de asimilación del consumismo (EAC)}

Esta escala consiste en una propuesta original que el nivel de incorporación de la necesidad de artículos - referenciados como icónicos del consumismo- cuyo valor simbólico es altamente apreciado en los procesos de identificación y pertenencia social. Los artículos considerados para esta construcción fueron: zapatos tenis; automóvil; motocicleta; ropa de salir; accesorios de vestir; teléfono inteligente; computadora; pantalla de televisión.

A cada uno de estos artículos se les atribuyen condiciones de innecesario, poco necesario, necesario y muy necesario. A partir de esta información a cada artículo se le aplica una escala aditiva compuesta, cuando el ítem se consideran de las primeras dos condiciones, la variable toma valor 0 y cuando se consideran la tercera y cuarta condición, se aplica un criterio de intensidad de asimilación. Para este último paso, se consultaron criterios de selección que los jóvenes tomarían en cuenta si tienen la 
oportunidad de comprar el ítem y que remiten a elementos de identificación simbólica que los mismos jóvenes consideran. Los criterios de selección se organizaron en las siguientes categorías: calidad; marca; originalidad; costo; moda: publicidad; prestigio; aprobación de pareja o amigo/a; otros.

Para cada ítem se le asigna valor 1 a cada criterio considerado, es decir, entre más criterios son considerados por los jóvenes asume mayor exigencia para cumplir con las condiciones consumistas de referencia. Sin embargo, se hizo una adecuación metodológica partiendo de que los criterios de exigencia fueron consultados con una pregunta abierta y se agruparon de acuerdo a estos nueve criterios precodificados; estos son los que se esperaba que los jóvenes consideran importantes al adquirir estos artículos, por tanto, no todos los jóvenes respondieron a la totalidad de criterios.

Con el fin de no definir un orden de criterios y excluir alguno, se decide ajustar la escala fijando un número mínimo de criterios que sea suficiente para captar la asimilación de consumismo. Los ítems tienen como valores medios 2 y 3 en su mayoría, por lo que se decide dar el máximo valor de la escala cuando un joven considere al menos 4 criterios de los 9 propuestos, sin importar cuales sean. Esto quiere decir que un joven con la máxima asimilación del consumismo es aquel que considera todos los artículos presentados indispensables y toma en cuenta al menos cuatro de los nueve criterios señalados.

Con esta distinción, se realiza un análisis de fiabilidad, se procede a generar la Escala de Asimilación del Consumismo y se recodifican los valores mayores a 32 en esta categoría (cuatro criterios para los nueve ítems).

\section{Análisis de confiablidad}

La Tabla 2 presenta el ejercicio de confiabilidad realizado con todos los estándares laborales de la subescala de precariedad laboral. En la primera columna se puede ver la lista de estándares considerados. En la segunda, el primer análisis realizado tomando en cuenta los 10 elementos. Se obtuvo un Alfa de 0.786, este valor se puede maximizar si se suprime el estándar de jornada laboral, se esperaría que llegue hasta 0.818 . La tercera columna de la Tabla 2 refleja ese procedimiento y se obtuvo un Alfa de $0.815 .{ }^{34}$ Aunque el análisis sugiere eliminar el estándar de horas extra para llegar a un índice de 0.833 , se decide dejar la escala con nueve valores y un alfa superior a 0.8 considerando este índice suficientemente confiable. Llevar ese indicador al máximo posible limita cada vez más el número de variables tomadas en cuenta, y se entiende que esto podría restar fuerza analítica con respecto a la propuesta teórica de la que se partió. 
Tabla 2

ANÁLISIS DE CONFIABILIDAD DE LA ESCALA DE EXCLUSIÓN/INCLUSIÓN SOCIAL

\begin{tabular}{lcc}
\hline Cantidad de elementos de la escala & 10 & 9 \\
\hline $\mathrm{N}$ & 173.69 & 174.82 \\
\hline Alfa de Cronbach con todos los elementos & 0.786 & 0.815 \\
\hline Alfa de Cronbach si el elemento se ha suprimido & & \\
Estabilidad laboral & 0.776 & 0.813 \\
Cumple jornada laboral & 0.818 & - \\
Salario mínimo & 0.782 & 0.814 \\
Forma de pago & 0.771 & 0.804 \\
Días por enfermedad & 0.750 & 0.790 \\
Vacaciones pagadas & 0.736 & 0.773 \\
Aguinaldo & 0.738 & 0.776 \\
Seguro de riesgo & 0.756 & 0.788 \\
Horas extra & 0.800 & 0.833 \\
Seguridad social & 0.733 & 0.769 \\
\hline
\end{tabular}

Fuente: Elaboración propia.

Así mismo, la Tabla 3 imprime el mismo ejercicio de confiabilidad para la escala de asimilación del consumismo. Destaca que se obtuvo el máximo valor posible para ese conjunto de variables con el primer procesamiento, un Alfa de 0.716, y el índice no puede ser mejorado eliminando algún ítem. Tomando en cuenta lo señalado anteriormente, se considera que la escala es suficientemente confiable para ser tomada en cuenta. ${ }^{35}$

Tabla 3

ANÁLISIS DE FIABILIDAD DE LA ESCALA DE ASIMILACIÓN DE CONSUMISMO

\begin{tabular}{lc}
\hline Cantidad de elementos de la escala & 8 \\
\hline $\mathrm{N}$ & 465.55 \\
\hline Alfa de Cronbach con todos los elementos & 0.716 \\
\hline Zapatos tenis & 0.673 \\
Automóvil & 0.701 \\
Motocicleta & 0.714 \\
Ropa de salir & 0.664 \\
Accesorios de vestir & 0.693 \\
Teléfono inteligente & 0.673 \\
Computadora & 0.692 \\
Pantalla de televisión & 0.690 \\
\hline
\end{tabular}

Fuente: Elaboración propia. 


\section{Ejercicio de Two Step Clusters}

La Tabla 4 detalla los resultados del análisis bietápico de conglomerados (Two-step clusters). Se muestran los pesos (porcentaje de casos que representa el conglomerado) y los centroides (medias de cada conglomerado en las escalas, el cruce de los ejes corresponde al centro del conglomerado) de cada tipo resultante.

Tabla 4

LOS GUIDO: DISTRIBUCIÓN Y CENTROIDES DE CADA CONGLOMERADO

\begin{tabular}{cccccccc}
\hline \multicolumn{3}{c}{ Distribución de clúster } & & \multicolumn{5}{c}{ Centroides } \\
\cline { 5 - 8 } Clúster & $\mathrm{N}$ & $\begin{array}{c}\text { \% de } \\
\text { combinado } \\
(\text { sin } \\
\text { ponderar })\end{array}$ & $\begin{array}{c}\% \\
\text { ponderado* }\end{array}$ & Media & $\begin{array}{c}\text { Consumismo } \\
\text { estándar }\end{array}$ & Media & $\begin{array}{c}\text { Eesviación } \\
\text { estándar }\end{array}$ \\
\hline 1 & 88 & 19.1 & 17.2 & 4.6982 & 0.75133 & 6.9206 & 1.01347 \\
2 & 88 & 19.1 & 20.4 & 2.7344 & 0.93480 & 4.8398 & 0.72677 \\
3 & 92 & 20.0 & 17.9 & 4.9490 & 0.82521 & 2.8250 & 1.11099 \\
4 & 39 & 8.5 & 8.7 & 7.8686 & 1.35624 & 7.0829 & 1.39767 \\
5 & 45 & 9.8 & 12.0 & 2.1875 & 0.97464 & 7.9463 & 0.86054 \\
6 & 30 & 6.5 & 7.0 & 8.2813 & 1.17143 & 2.4135 & 0.97366 \\
7 & 79 & 17.1 & 16.9 & 2.1123 & 0.80875 & 1.9650 & 0.85769 \\
\hline Combinado & $461^{* *}$ & 100 & 100 & 4.1866 & 2.18849 & 4.6774 & 2.39638 \\
\hline
\end{tabular}

Fuente: Elaboración propia. ${ }^{*}$ Se aplicaron los factores de ponderación. ${ }^{* *} E l$ ejercicio excluyó automáticamente 6 casos de los 467 totales.

Esta información sirvió para ubicar los casos en un plano cartesiano, en el eje $x$ se ubica la escala de asimilación del consumismo y en el eje y la escala de exclusión/ inclusión; ambos ejes se mueven entre valores 0 y 10. Sobre este plano se ubican los conglomerados, de acuerdo a los respectivos centroides; cada conglomerado es incorporado en una "burbuja" cuyo tamaño representa el porcentaje de casos que contiene cada uno.

\section{Notas}

1 El presente artículo se basa en los principales hallazgos de la tesis de licenciatura intitulada: Análisis de las relaciones entre exclusión social, violencias y consumismo en jóvenes de Los Guido de Desamparados (Hernández Ulloa). Fue desarrollada en el marco del proyecto Entre la violencia y 
el empleo. Los dilemas de jóvenes de comunidades urbanas de Centroamérica realizado por FLACSO Costa Rica y FLACSO El Salvador, con el auspicio del International Development Research Centre (IDRC) de Canadá. La información sobre el proyecto (equipo de investigación, objetivos, metodología, informes de resultados, etc.) y la versión digital de la tesis se pueden consultar en: http://flacso.or.cr/index.php/areas-de-trabajo/proyectos-institucionales/proyecto-deinvestigacion-entre-la-violencia-y-el-empleo-los-dilemas-de-jovenes-de-comunidadesurbanas-marginales-en-centroamerica

Se agradecen los comentarios recibidos por parte del Dr. Juan Pablo Pérez Sáinz al borrador de este artículo.

Pérez Sáinz señala que estamos ante una individualización que no logra aportar a la relativización de las desigualdades; además, no ha logrado generar un nuevo orden social donde el consumo desplace la producción: "estamos ante una situación paradójica. Por un lado, el orden (neo) liberal potenció y celebró al máximo el individualismo como nunca antes en la historia de la región (y lo cifró en el consumismo). Pero, por otro lado, los soportes que generó para los procesos de individualización son frágiles e inestables. Así, el mercantilizar el sistema de seguridad social, propició individualización, pero erosionó su soporte: la vieja ciudadanía social" (Pérez Sáinz 210).

Para Douglas e Isherwood (19) el consumo tiene un anclaje importante en el campo cultural, esto se debe en parte a que las mercancías -como señales de las categorías racionales- contienen consigo ciertos significados que son interpretados y reproducidos en el campo de la cultura, así: "en el interminable diálogo sobre el valor que está implícito en el acto del consumo, las mercancías en su conjunto representan una serie más o menos coherente y deliberada de significados, que podrán ser percibidos solo por quienes conozcan el código y los escudriñen en busca de información".

Diversas investigaciones han señalado que la exclusión social afecta a las sociedades centroamericanas, particularmente a las comunidades que se encuentran en los márgenes urbanos. Se conoce la afectación en términos de acceso a los aspectos básicos que deben ser garantizados por los Estados, también se reconoce que estos territorios afrontan múltiples formas de violencia y que estos problemas tienen un impacto diferenciado según los grados de exclusión o inclusión social en cada comunidad. Las investigaciones concluyen que la exclusión social forma parte de una dinámica que refuerza desigualdades a lo interno de territorios urbanos y que la salida a través de la inserción laboral es posible, pero difícil de alcanzar para quienes habitan estos territorios debido a los obstáculos estructurales que padecen sistemáticamente (Savenije y Andrade-Eekhoff; Beirute-Brealey; Pérez Sáinz, 2012; Pérez Sáinz, 2015).

Acercarse a la población joven en contextos urbanos conlleva una reflexión sobre la heterogeneidad de juventudes que coexisten en esos espacios. Para efectos de este texto, reportaremos la decisión metodológica con la que se definió el criterio de juventud, esto fue desde un punto de vista etario, delimitando como personas jóvenes a quienes tiene entre 18 y 29 años de edad. Para profundizar el análisis sobre las actitudes hacia el futuro de jóvenes de Los Guido con respecto a tres dimensiones (imaginación de la nación, estigmatización y desempleo oculto) se puede consultar: Pérez Sáinz y Hernández Ulloa.

Se ha elaborado una tipología, siguiendo lo planteado por Weber, como un ejercicio de elaboración conceptual que permite expresar características fundamentales presentes en las estructuras en la sociedad, y que no necesariamente aparecerán como una versión que refleja una forma "absolutamente pura" de este constructo conceptual. Esto es un ejercicio de reconstrucción analítica de la realidad para lograr su comprensión. Weber afirma que "para 
que con estas palabras [los conceptos] se exprese algo unívoco, la sociología debe formar, por su parte, tipos puros (ideales) de esas estructuras, que muestren en sí la unidad más consecuente de una adecuación de sentido lo más plena posible; siendo por eso mismo tan poco frecuente quizá en la realidad -en la forma pura absolutamente ideal del tipo- como una reacción física calculada sobre el supuesto de un espacio absolutamente vacío. Ahora bien, la casuística sociológica solo puede construirse a partir de estos tipos puros (ideales)" (Weber 17).

Un aspecto medular para la consolidación del consumismo como generador de identidad fue influencia de la publicidad. Naomi Klein, señala que, en Estados Unidos, luego la crisis de identidad de las marcas con la generación del babyboom, se dio un reauge de estas durante el último decenio del siglo pasado. El marketing volvió la mirada a un sector demográfico que había pasado desapercibido: la juventud. Al respecto señala: “No era época para vender Tide y Snuggle [productos de limpieza] a las amas de casa, sino de lanzar la MTV, Nike, Hilfilger, Microsoft, Netscape y Wired a los adolescentes de todo el mundo y a sus imitadores. Sus padres podían haber cuidado su dinero, pero los hijos estaban dispuestos a pagar para ser aceptados. Por medio de este proceso, la presión de los coetáneos se convertía en una poderosa fuerza del mercado que dejaba pálido el consumismo de los padres" (Klein 87). Este efecto de la publicidad también ha sido señalado por diversos autores para el caso de América Latina, en particular el costarricense (Vega Jiménez; Viales-Hurtado; Calderón-Umaña).

Con respecto a la consolidación de espacios de consumo en territorios urbanos costarricense, un estudio de caso sobre el mall Lincoln Plaza en Moravia, evidencia el proceso de territorialización que mueven actores empresariales para ubicar los espacios de consumo como nuevos nodos territoriales que articulan la realidad social. El estudio concluye que "el mall puede interpretarse como un territorio "esponja", ya que absorbe elementos de su contexto geográfico: actividades, relaciones, hitos históricos, infraestructura, imágenes y naturaleza, hasta su conveniencia o saturación. Estos son elementos que se apropian y se controlan, según sus intereses y objetivos, para presentarlos de una forma distinta dentro de su espacio. Lo anterior permite que se construya una geografía que tiene como intención, generar las condiciones para propiciar un consumo, lo cual se enmascara con condiciones físicas estilizadas para que las personas se introduzcan y permanezcan en su espacio" (JiménezCorrales 173).

10 Lungo-Rodríguez ha señalado para el caso salvadoreño como las nuevas elites que surgen de sectores medios desde finales de los años noventa se han caracterizado, entre otras cosas, por dinámicas privativas y de exclusividad asociadas al consumo. Esto forma parte de los repertorios de legitimación de las desigualdades. Al respecto la autora señala de forma concluyente que, para estos sectores "la sociabilidad tiene lugares en espacios físicos circunscriptos, entre los que destacan los lugares de consumo exclusivos y excluyentes. Este conjunto de prácticas [junto con el carácter endogámico reforzado por la escuela, el trabajo y la familia] tiende a limitar la interacción social y el contacto con personas de los grupos subalternos, mientras fortalece la creación de territorios sociales diferenciados" (Lungo-Rodríguez 269).

11 Respecto al cambio gestado por el MCC, Torres-Rivas señala que en la década del sesenta el comercio internacional tiene condiciones cualitativamente distintas a las de la década de los cincuenta: importaciones, comercio internacional, cambios en las economías de los países desarrollados, rasgos monopólicos y expansión supranacional. Estas novedosas características generaron nuevos niveles de acumulación capitalista cuya consecuencia fue la tendencia integradora del capital financiero regional (Torres-Rivas). 
Las tarjetas de crédito han sido uno de los productos financieros que están asociados al consumo suntuario, las tarjetas por si mismas también se han presentado como un bien a consumir por el prestigio que puede generar. Resulta sorprendente la presencia que tienen en la sociedad costarricense, según el Ministerio de Economía, Industria y Comercio, el primer trimestre del 2016 en Costa Rica había 453 tipos de tarjetas de crédito, para un total de 2252693 tarjetas de crédito en circulación (73.6 \% de estas tiene tasas de interés anual entre $40 \%$ y 50 \%) para un total de 896719 millones de colones de saldo de deuda (Hidalgo Portilla y Porras Alvarado).

En Costa Rica varias manifestaciones de violencia tienen un impacto significativo en población juvenil. $45 \%$ de las víctimas de homicidios dolosos en el 2016 fueron jóvenes entre 15 y 29 años (Oficina de Planes y Operaciones). El 89 \% de la población joven ha experimentado violencia en el hogar; mientras que el 33\% de las personas jóvenes considera que el principal problema del país que les afecta es la inseguridad ciudadana (Consejo Nacional de la Política Pública de la Persona Joven). A su vez, a nivel local, el 82.1 \% de jóvenes de Desamparados identifican la inseguridad ciudadana como principal problema del cantón, mientras que el $74.7 \%$ de jóvenes señalan al menos una situación de discriminación en el municipio, de las cuales $41.6 \%$ se da por la condición socioeconómica y 37.8 \% por la condición de joven (CPJ y UNFPA).

14 El en el caso costarricense, se trata de un fenómeno de pandillas que dista por completo de lo que acontece en países del norte de Centroamérica. Estas, más conocidas como maras, están categorizadas por la OEA como pandillas violentas, mientras aquellas corresponden a pandillas transgresoras, un fenómeno de menor escala. Según esta famosa categorización, las segundas "surgen en contextos de exclusión y pobreza estructural... como un intento por satisfacer sus derechos de supervivencia, protección y participación, organizándose sin supervisión y desarrollando sus propias normas y criterios de membresía, afianzando una territorialidad y una simbología que da identidad y se consolida con la rivalidad y el enfrentamiento permanente con las pandillas enemigas" (OEA 67-68).

Se conoce como "búnker" a distintos tipos de locales (casas, comercios, casas abandonadas, entre otros) en los que se almacena y distribuye drogas, principalmente para narcomenudeo.

En el cantón de Desamparados la incidencia delictiva en general se ha presentado como una problemática en auge, por ejemplo, en los años 2012 y 2013 el cantón ocupó el cuarto lugar entre los que más asaltos reportaron; fue el tercero que más reportó robo de vehículos; el quinto en reportes de asalto a viviendas; en violaciones sexuales fue cuarto en el 2012 y sexto en 2013; y ocupó el cuarto lugar en homicidios dolosos en el 2013 (Oficina de Planes y Operaciones).

Como ya hemos señalado al inicio del artículo, este se basa en los resultados de la tesis de licenciatura, dicha investigación consta de dos fases, la fase cuantitativa sobre la cual se desarrolla este artículo, y la fase cualitativa. Para profundizar sobre los resultados de la segunda fase véase (Hernández Ulloa).

La encuesta a la que se hace referencia se realizó entre el 20 de octubre y el 20 de diciembre del 2016. A final del artículo se incorpora un breve anexo metodológico en el que detallan aspectos específicos de la muestra; la construcción metodológica de las escalas empleadas en el análisis de conglomerados; y los análisis de confiabilidad aplicados a este ejercicio.

19 Para la elaboración del diseño muestral se contó con la colaboración de Gilbert Brenes Camacho, investigador del Centro Centroamericano de Población y docente de la Escuela de Estadística de la UCR, los detalles sobre el proceso de muestreo se pueden consultar 
en el anexo metodológico. Una revisión más a profundidad de se encuentra en Hernández Ulloa, 189-192.

Para sobrepasar esta dificultad Pérez Sáinz $(2012,2015)$ utiliza una "escala de fuentes de ingresos" que asigna valores de acuerdo al tipo de ingreso del hogar donde todos los miembros son inactivos, en nuestro caso, no se recupera la información de fuentes de ingresos del hogar, únicamente las del joven en el caso que corresponda.

Cuando fue necesario, se aplicó homologación de las subescalas, es decir, se convirtieron los valores propios de cada subescala -valor mínimo y máximo dictado por los rangos de las variables- en una escala equivalente con valores mínimo y máximo establecidos (usualmente generando un rango entre 0 y 10) sin perder la estructura o magnitud de la misma. Esto permitió la compactación de las diferentes subescalas en una sola. La fórmula empleada fue: valor homologado $=(($ valor de escala - valor mínimo $) /($ valor máximo-valor mínimo $)){ }^{*} 10$ (Pérez Sáinz, 2012, 105).

En este punto se realizó una adecuación metodológica importante. Los criterios de selección fueron consultados con una pregunta abierta y se agruparon de acuerdo a estos nueve criterios precodificados; estos son los que se esperaba que los jóvenes consideran importantes al adquirir estos artículos, por tanto, no todos los jóvenes respondieron a la totalidad de criterios. Se decide ajustar la escala fijando un número mínimo de criterios que sea suficiente para captar la asimilación de consumismo, el máximo valor de la escala se asigna cuando una persona joven considere al menos 4 criterios de los 9 propuestos, sin importar cuales sean. Es decir, la máxima asimilación del consumismo está signada por quienes consideran todos los artículos presentados indispensables y toma en cuenta al menos cuatro de los nueve criterios señalados.

El ajuste en la operacionalización de las problemáticas juega un papel clave en la construcción de este tipo de escalas, ya que es ahí donde se lograr vaciar la propuesta analítica en las herramientas de recolección de información. Este ejercicio incide en la posibilidad de lograr constructos analíticos que tengan mejores resultados en este tipo de análisis. Este aspecto resulta fundamental para tomar en cuenta para futuras investigaciones en las que se quiere profundizar sobre la problemática del consumismo empleando análisis cuantitativos.

Otros datos de la encuesta refuerzan esta problemática. Quizás el más revelador tiene que ver con la problemática del desempleo. Se calculó una tasa de desempleo abierto para estas personas jóvenes es de $16.9 \%$. Esto se agrava cuando se toman en cuenta a la población inactiva que señaló disponibilidad para trabajar si se diera la oportunidad, pues la tasa se eleva al $50.0 \%$. Aquí el componente de género tiene relevancia: de las mujeres recluidas en la lógica doméstica (42.8 \% de las mujeres jóvenes del distrito que señalaron como condición de actividad "solo quehaceres en el hogar") el $76.5 \%$ señaló disponibilidad para ingresar al mercado laboral.

Según los de la encuesta, $55.0 \%$ de las personas jóvenes de Los Guido han abandonado los estudios (antes de concluir la secundaria), las razones de abandono tienen una diferencia de género, la mayoría de hombres (23.6\%) lo hizo por compromisos laborales, mientras que la mayoría de mujeres $(28.1 \%)$ por compromisos familiares. Más información sobre estas características se encuentra en el texto completo de la tesis.

26 En este mismo sentido los hallazgos de Beirute-Brealey confirman que la vivencia de la juventud en la exclusión social está asociada con una vida cargada de desmotivación y ausente de perspectiva a futuro: "para estos muchachos no existe brecha entre las aspiraciones y 
expectativas, simplemente porque éstas no existen. Para ellos "la vida se vive en el presente", en sus necesidades de ahora y no en lo que puede ser que les depare el futuro. Pareciera que no conciben un futuro propio al que deben moldear; la frase frecuente en su discurso respecto a las diversas dimensiones fue: "lo que salga"" (Beirute-Brealey 79).

Los hallazgos de la fase cualitativa de esta investigación revelan que en este tipo de jóvenes no existen grupos de referencia en el territorio, ni participación a través de redes sociales, generando una suerte de aislamiento que le auto excluye de dinámicas consumistas, pues en definitiva no se revela ningún interés de participar en esas dinámicas (Hernández Ulloa 131).

28 Estas hipótesis se basan en los resultados obtenidos al preguntar sobre posibles mecanismos que emplearían estos jóvenes para acceder a los bienes de consumo consultados en caso de no tener recursos. El desarrollo de esta información se encuentra en el cuarto capítulo de la tesis (Hernández Ulloa 110-113).

Cada estrato se posestratificó según el sexo del entrevistado (hombres o mujeres) y según el grupo de edad (de 18 a 24 años y de 25 a 29 años), se tomaron en cuenta los seis estratos y las cuotas sirvieron para corregir representatividad a través de factores de ponderación en el análisis.

Resulta valioso para esta construcción la propuesta analítica y metodológica que aporta MoraSalas en términos de la medición de la precariedad laboral.

31 En la pregunta del cuestionario se utilizó como referencia el salario Mínimo Minimorun, que según el Ministerio de Trabajo y Seguridad Social para el II semestre del 2016 equivalía a CRC 251239.04 .

Resaltar la aclaración del aseguramiento por trabajo, pues es la modalidad de seguro social que se captó en el cuestionario. Además, se conoce la existencia de otros mecanismos de aseguramiento que no pasan por los mercados de trabajo, sino que son modalidades de asistencia estatal para poblaciones vulnerables, sin embargo, por el carácter de la unidad de análisis no se indagaron.

33 Cuando fue necesario, se aplicó homologación de las subescalas, es decir, se convirtieron los valores propios de cada subescala -valor mínimo y máximo dictado por los rangos de las variables- en una escala equivalente con valores mínimo y máximo establecidos (usualmente generando un rango entre 0 y 10) sin perder la estructura o magnitud de la misma. Esto permitió la compactación de las diferentes subescalas en una sola. La fórmula empleada fue: valor homologado $=(($ valor de escala - valor mínimo $) /($ valor máximo-valor mínimo $))$ * 10 (Pérez Sáinz 2012, 105).

El valor obtenido (.815) al quitar el ítem sugerido difiere del valor esperado (.818) debido a un valor perdido en el ítem de jornada laboral, por lo tanto, la $\mathrm{N}$ del segundo procesamiento es más pequeña (173.69) que la $\mathrm{N}$ del primer procesamiento $(174,82)$. Los valores no enteros de las $\mathrm{N}$ se deben al redondeo por el uso de factores de ponderación.

El ajuste en la operacionalización de las problemáticas juega un papel clave en la construcción de este tipo de escalas, ya que es ahí donde se lograr vaciar la propuesta analítica en las herramientas de recolección de información. Este ejercicio incide en la posibilidad de lograr constructos analíticos que tengan mejores resultados en este tipo de análisis. Este aspecto se debe tomar en cuenta para futuras investigaciones en las que se quiere profundizar sobre la problemática del consumismo empleando análisis cuantitativos. 


\section{Bibliografía}

Baker, Andy. The market and the masses in Latin America. Policy reform and consumption in liberalizing economies. Cambridge University Press, 2009.

Bauman, Zygmunt. Vida de consumo. Fondo de Cultura Económica, 2007.

Beirute-Brealey, Tatiana. ¿Con qué soñás? Expectativas y aspiraciones sobre el futuro de jóvenes urbanos costarricenses. FLACSO, 2012.

Calderón-Umaña, Rodolfo. Delito y cambio social en Costa Rica. FLACSO, 2012.

Consejo Nacional de la Política Pública de la Persona Joven. Segunda encuesta nacional de juventudes: informe de principales resultados. Consejo Nacional de la Política Pública de la Persona Joven, 2013.

CPJ y UNFPA. Encuesta sobre la realidad de las personas jóvenes de Desamparados. Programa Conjunto Juventud, Empleo y Migración, 2011. http://cpj.go.cr/migracion/docs/JEM/ encuestas/Desamparados/BoletinDesamparados.pdf

Douglas, Mary Tew y Baron C. Isherwood. El mundo de los bienes: hacia una antropología del consumo. Trad. Enrique Mercado, Grijalbo, Consejo Nacional para las Cultura y las Artes, Dirección General de Publicaciones, 1990.

García-Canclini, Néstor. Consumidores y ciudadanos: conflictos multiculturales de la globalización. Grijalbo, 1997.

Hernández Ulloa, Francisco. Análisis de las relaciones entre exclusión social, violencias y consumismo en jóvenes de Los Guido de Desamparados. Universidad de Costa Rica, 2017. Recuperado de http://flacso.or.cr/images/documentos/tesis-noviembre-exclusionsocial-joveneslosguido.pdf

Hernández Ulloa, Francisco y Juan Pablo Pérez Sáinz. “Violencias, territorio y mediaciones sociales. Retos de jóvenes de Los Guido para salir adelante en la vida". Vidas sitiadas. Jóvenes, exclusión laboral y violencia urbana en Centroamérica. Ed. Juan Pablo Pérez Sáinz, FLACSO; IDRC, 2018, http://www.flacso.or.cr/images/docs_proyectos/librovidasitiadasweb.pdf

Hidalgo Portilla, Jorge y Luisiana Porras Alvarado. Primer estudio trimestral de tarjetas de crédito del 2016. DAEM-INF-002-16, Ministerio de Economía, Industria y Comercio, 2016. http:// reventazon.meic.go.cr/informacion/estudios/2016/tarjetas/enero/credito.pdf

Jiménez-Corrales, Andrés. Cartografía de la territorialización del mall Lincoln Plaza. Moravia, Costa Rica 2005-2016. Universidad de Costa Rica, 2016.

Klein, Naomi. No logo: el poder de las marcas. Trad. Alejandro Jockl, Paidós, 2001.

León Araya, Andrés. "Algunos apuntes históricos sobre el sector financiero costarricense (1985-2007)". Anuario de Estudios Centroamericanos, vol. 38(2012): 213-249.

Lungo-Rodríguez, Irene. "Nosotros, educados y emprendedores". Legitimación de privilegios socioeconómicos en clases medias altas en El Salvador. El Colegio de México, 2017.

Mata, Esteban. "25.000 pobladores de Los Guido están condenados a vivir como precaristas". La Nación, 2014, http://www.nacion.com/nacional/gobierno/habitantes-Guido-condenados-vivir-precario_0_1426657345.html

Mora-Salas, Minor y Juan Pablo Pérez Sáinz. Se acabó la Pura Vida. Amenazas y desafíos sociales en la Costa Rica del Siglo XXI. FLACSO, 2009.

Mora-Salas, Minor y Franklin Solano Castro. Segregación urbana en el área metropolitana de San José: el caso de los nuevos asentamientos urbanos 1980-1990. Universidad de Costa Rica, 1992. 
Mora-Salas, Minor. "La medición de la precariedad laboral: problemas metodológicos y alternativas de solución". Revista Trabajo, 5.9(2012): 89-124.

OEA. Definición y categorización de pandillas. Secretaria General de la Organización de Estados Americanos, junio 2007. https://www.oas.org/dsp/documentos/pandillas/informe. definicion.pandillas.pdf

Oficina de Planes y Operaciones. Memoria Anual del OIJ 2016. Organismo de Investigación Judicial, 2017. https://www.poder-judicial.go.cr/oij/index.php/component/phocadownload/category/34-memoria-institucional-oij?download=1060:memoria-institucional-oij-2016

Pérez Sáinz, Juan Pablo, Ed. Sociedades Fracturadas: la exclusión social en Centroamérica. FLACSO, 2012.

Pérez Sáinz, Juan Pablo. Mercados y Bárbaros. La persistencia de las desigualdades de excedente en América Latina. FLACSO, 2014.

Pérez Sáinz, Juan Pablo. Ed. Exclusión social y violencias en territorios urbanos centroamericanos. UKaid; IDRC/ CDRI; FLACSO, 2015.

Pérez Sáinz, Juan Pablo. Una historia de la desigualdad en América Latina: la barbarie de los mercados desde el siglo xix hasta hoy. Siglo XXI Editores, 2016.

Pérez Sáinz, Juan Pablo y Francisco Hernández Ulloa. Violencias y exclusión social. Retos de jóvenes de Los Guido para salir adelante en la vida. FLACSO-Costa Rica/IDRC, 2017.

Pérez Sáinz, Juan Pablo y Francisco Hernández Ulloa. “Entre el optimismo y el pesimismo. Jóvenes de asentamientos urbanos populares en Centroamérica". CariCen, 8(2018): 25-40.

Pérez Sáinz, Juan Pablo y Minor Mora Salas. "Exclusión social, desigualdades y excedente laboral. Reflexiones analíticas sobre América Latina". Revista Mexicana de Sociología, 68.3 (2006): 431-65.

Pérez Sáinz, Juan Pablo y Minor Mora Salas. La persistencia de la miseria en Centroamérica. Una mirada desde la exclusión social. FLACSO, 2007.

Portes, Alejandro y Bryan R. Roberts. “Introducción. La ciudad bajo el libre mercado. La urbanización en América Latina durante los años del experimento neoliberal". Ciudades latinoamericanas: un análisis comparativo en el umbral del nuevo siglo. Eds. A. Portes et al. Prometeo Libros, 2005. http://meme.phpwebhosting.com/ migracion/rimd/coleccion_america_latina/ciudades_latinoamericanas/introduccion.pdf

Presidente de la República, et al. Crea el distrito Los Guido, trece del cantón Desamparados. Decreto Ejecutivo, 31380-G, 1 Sept. 2003, http://www.pgrweb.go.cr/scij/Busqueda/ Normativa/Normas/nrm_texto_completo.aspx?param1=NRTC\&nValor1=1\&nValor2= 51821\&nValor3=56173\&strTipM=TC\#ddown

Robles Rivera, Francisco. “Los de entonces ya no son los mismos. Acumulación por desposesión en la última década en El Salvador y Costa Rica". Anuario de Estudios Centroamericanos, 37(2011):105-37.

Savenije, Wim y Katharine Andrade-Eekhoff. Conviviendo en la orilla: exclusión social y violencia en el área metropolitana de San Salvador. FLACSO, 2003.

Segura, Ramiro. "El espacio urbano y la (re) producción de desigualdades sociales". desiguAldades.net, 65(2014). http://www.diss.fu-berlin.de/docs/receive/FUDOCS_document_000000020172

Torres-Rivas, Edelberto. Interpretación del desarrollo social centroamericano. FLACSO, 1989. 
Vega Jiménez, Patricia. "Evolución de la historia del consumo (una discusión inconclusa)". Consumidores: apuntes para el debate actual y perspectivas para la cuidadania contemporánea. Eds. J. Guzmán y L. E. Jiménez. Friedrich-Ebert-Stiftung, 2004. 9-32.

Viales-Hurtado, Ronny. "La historia del consumo. Una propuesta de enfoque para el caso de América Central entre 1850 y 1950". Tendencias del consumo en Mesoamérica. Ed. J. J. Marín y P. Vega. Editorial UCR, 2008. 23-36.

Villamichel-Morales, Pablo. "Análisis de los 20 años de competencia en el sector bancario costarricense". Ponencia para Vigesimoprimer informe Estado de la Nación en Desarrollo Humano Sostenible 2014, Mayo 2015. https://estadonacion.or.cr/files/biblioteca_virtual/021/economia/VillamichelBancaC3.pdf

Weber, Max. "Conceptos sociológicos fundamentales". Economía y sociedad. Esbozo de sociología comprensiva. Fondo De Cultura Económica, 2002. 1-45.

Zúñiga-Ramírez, Cesar. "Reforma del Estado y sistema financiero de Costa Rica: de la liberalización a la globalización". Revista Parlamentaria, 22.1(2016): 79-114.

Francisco Hernández Ulloa. Costarricense. Licenciado en Sociología por la Universidad de Costa Rica. Investigador de FLACSO-Sede académica Costa Rica, actualmente coordina el Diplomado Centroamericano de Seguridad Ciudadana y Prevención de la Violencia. Profesor en la Escuela de Sociología de la Universidad de Costa Rica.

Contacto: fhernandez@flacso.or.cr

ORCID: 0000-0001-9242-8191 